\title{
Design and Development of Microcontroller Based Auto- Flow Assembly for Biosensor Application
}

\author{
Shiwani Saini \\ School of Engineering \& Technology \\ Sharda University \\ Greater Noida, India
}

\author{
C.R.Suri \\ Institute of Microbial Technology (IMTECH) \\ Sector 39, Chandigarh \\ India
}

\begin{abstract}
An automated flow assembly for a biosensor has been developed in this paper. In recent years there has been a growing interest in the field of in-vivo biosensors for continuous monitoring. For this continuous monitoring of biological interactions, a need for the development of auto-flow assembly arises. The automated flow assembly inherently coupled to a biosensor, forms a Flow Injection Analysis (FIA) system. The designed auto-flow assembly consists of three micro-fluidic solenoid valves actuated through a microcontroller AT89C51. A programmable turn-on time for each valve can be entered through a $4 \times 3$ keypad and its switching can be controlled by the microcontroller. In addition, a temperature-sensing unit for online monitoring of the buffer temperature at the biosensor has been interfaced to the microcontroller. The automated flow assembly offers the advantage of faster response. The work includes interfacing of the temperature sensor, keypad (for selecting the time), display unit (liquid crystal display) and solenoid valves to the microcontroller and development of the software for the same.
\end{abstract}

\section{General Terms}

Microcotrollers, automated flow assembly

\section{Keywords}

Microcontroller, biosensor, solenoid valves, flow-assembly, flow injection analysis

\section{INTRODUCTION}

A biosensor is a device that incorporates a biological sensing element either intimately connected to or integrated within a transducer.

\begin{abstract}
The usual aim is to produce a digital electronic signal, which is proportional to the concentration of a specific chemical or a set of chemicals. In recent years great amount of work has ensued in the field of biosensors as they offer great opportunities in fields like medicine, environment and defence.

Flow assembly is an experimental setup to allow an analyte in gaseous or liquid form to flow through a flow cell onto a biosensor (detector). Since most biosensors for the detection of biological interactions require the analyte to be in liquid or gaseous form, a flow through set up is desired to pump the analyte/sample. The block diagram representation of a flow through set up is shown in figure 1.
\end{abstract}

The earlier flow assemblies utilized a manually operated system as shown in figure 2. This manual flow arrangement consists of a pumping mechanism, multiposition valves, reactors/mixing chambers, display unit and a detector. A pump is combined with a directional valve and the reagent and sample are injected sequentially through the manually operated multiposition selection valve. The sample and the reagent then form a detectable specie to produce a quantifiable signal that can be detected by the detector.

Manual arrangements for delivering the sample and reagents through the flow cell [1] and [2] have been reported. The system used a sampler which permitted insertion of sample after suitable time interval by a multiposition valve. A miniaturized fiber-optic biosensor operating on the principles of FIA was reported by Scheper and Buckman [3]. It utilized two independent sample loops to inject the sample and a timer system to control the magnetic valves. Simonian [4], proposed an amperometric biosensor for L-tryptophan determination, which consisted of a flow injection system coupled to an amperometric biosensor. It

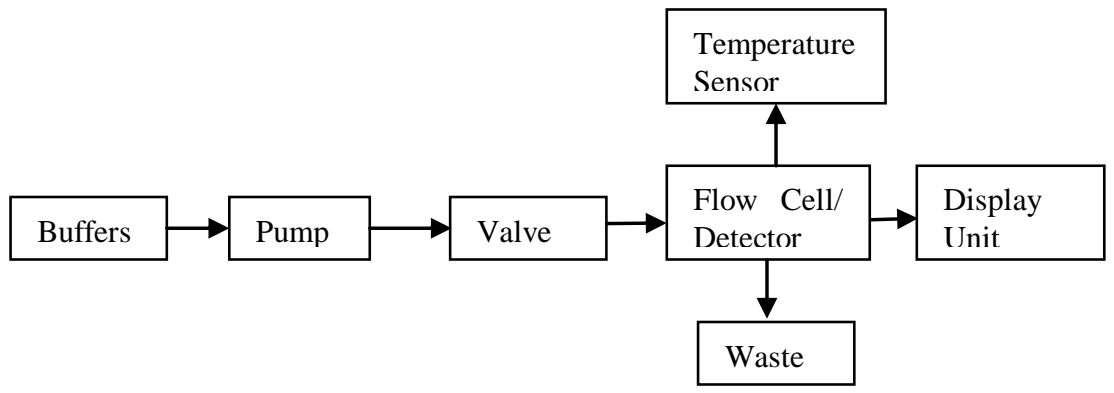

Figure 1 Schematic representation of a flow assembly 
utilized a temperature controlled flow-through column to inject the substrate on to the biocatalyst. The signal was processed using a computer. Flow through systems for direct controlling of valves and monitoring of the data through a $\mathrm{PC}$ have been reported [4], [5], [6],[7] and [8].

The main aim of the present work is to develop an automated flow assembly in place of a manually operated flow assembly. The automation is carried out with the help of a microcontroller.

For most immunobiosensor applications, three different buffers, a washing buffer, a binding buffer and an elution buffer are required. Elution buffer equilibrates the flow cell, binding buffer binds the biological molecule onto the detector and washing buffer washes the flow cell to remove the traces of the buffers used earlier. Each of these buffers requires a separate valve to pump the buffer through the flow cell.

The purpose of this automated flow assembly is to pump these buffers through the flow cell containing the detector/biosensor sequentially. The volume of buffers to be passed through the flow assembly can be controlled by selecting the duration for which a specific valve remains turned on. This actuation is controlled by the microcontroller AT89C51. The precise time for each valve to remain switched on is entered through a $4 \times 3$ keypad. A temperature sensor AD590 has been interfaced to the microcontroller through an analog to digital converter (ADC) ICL7109 for the purpose of monitoring the temperature of each buffer. A 16x2 characters alphanumeric liquid crystal display (LCD) unit is used to display both, time delay for each valve and the temperature of the analyte. unit (ALU), PC (program counter), SP (stack pointer), registers and in addition features like RAM, ROM, parallel input/output, serial input/output, counters and clock circuit. Like a microprocessor, a microcontroller is a general-purpose device, but one that is meant to read data, perform limited calculations on that data and control its environment based on those calculations. The prime use of a microcontroller is to control the operation of a machine using a fixed program stored in the ROM that does not change over a lifetime of a system. For the purpose of automating the flow assembly, an Atmel AT89C51 microcontroller is used. It is a low power, high performance CMOS, 8-bit, 40-pin microcontroller with $4 \mathrm{~K}$ bytes of flash programmable and erasable read only memory (EPROM). The on chip flash allows the program memory to be rapidly reprogrammed using a non-volatile memory programmer.

\subsection{Peristaltic Pump}

Liquid pumps maintain the flow of liquid through the valve. A peristaltic pump has been used in this flow assembly as it offers the advantage of the use of silicon/PTFE tubing as the pressure chamber. As a result, the fluid does not contaminate the pump; cleanup requires only change of tubing. In addition the pump offers excellent repeatability in low volume dispensing and metering applications. The valve less design eliminates clogging and siphoning of liquid under most conditions. Moreover, reversibility of flow has an added advantage. The peristaltic pump has a flow rate ranging from $1.5 \mathrm{~mL} / \mathrm{hr}$ to $156.5 \mathrm{~mL} / \mathrm{hr}$ and operates conveniently at a pressure of up to $100 \mathrm{psi}$. It has the advantage of very low power consumption as the current rating of the pump is $100 \mathrm{~mA}$.

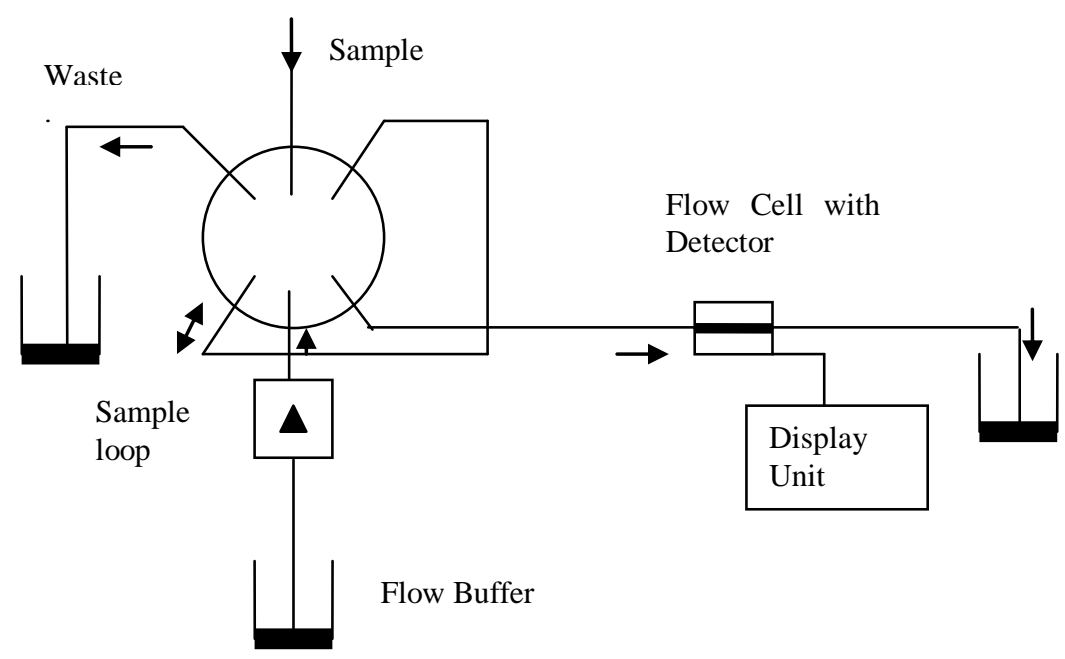

Figure 2 Manually operated flow assembly

\section{MATERIALS AND METHODS}

The flow assembly required the following basic components

\subsection{Microcontroller}

Microcontroller is a microprocessor whose design incorporates all the features of a microprocessor that is an arithmetic and logic

\subsection{Micro Fluidic Solenoid Valves}

Valves are the apparatus designed to maintain, restrict, or meter the flow of materials through pipes, hoses, tubing or entire systems. For this flow assembly, solenoid valves have been used since they have corrosion resistant all plastic body and viton 
seals. This makes them ideal for use with corrosive and aggressive chemicals. Their operating temperature is in the ambient temperature range $\left(4-65^{\circ} \mathrm{C}\right)$ and pressure requirements are also low (up to $40 \mathrm{psi}$ ). These valves can have continuous duty cycle and require only a few milliseconds to energize. Their typical response time is $15-30 \mathrm{~ms}$ from inactive to active stage and 5-10 ms from active to inactive stage. They operate on $3 \mathrm{~V}$ $\mathrm{DC}, 400 \mathrm{~mA}$ and their typical power consumption is $1.2 \mathrm{~W}$ which is very low.

\subsection{Display Unit}

The display unit used is a $16 \times 2$ (16 characters, 2 lines) alphanumeric liquid crystal display (LCD), which can be interfaced with a 4-bit or 8-bit microprocessor or microcontroller. Liquid crystal display is preferred since the use of CMOS LCD controller and driver ICs results in low power consumption. Since LCDs consume less power, they are compatible with low power electronic circuits, and can be powered for long duration. Moreover LCDs are lightweight, have long life and wide temperature range.

\subsection{Temperature Sensor}

A temperature sensor $\mathrm{AD} 590$ is used to measure the temperature of the analyte. It is a two terminal integrated temperature transducer that produces an output current proportional to absolute temperature. For supply voltages between $+4 \mathrm{~V}$ and +30 $\mathrm{V}$, the device acts as high impedance, constant current regulator passing $1 \mu \mathrm{A} / \mathrm{K}$. It has a calibration accuracy of $0.5^{\circ} \mathrm{C}$ and linearity of $0.3^{\circ} \mathrm{C}$. Laser trimming of the chip's thin film resistors is used to calibrate the device to $298.2 \mu \mathrm{A}$ output at $298.2 \mathrm{~K}$ (25 $\left.{ }^{\circ} \mathrm{C}\right)$. AD590 can be used in any temperature sensing application between $-55^{\circ} \mathrm{C}$ to $+150{ }^{\circ} \mathrm{C}$ in which conventional electrical temperature sensors are currently employed. Linearization circuitry, precision voltage amplifiers, resistance measuring circuitry and cold junction compensation are not needed in applying the AD590. The AD590 is available in chip form, making it suitable for hybrid circuits and fast temperature measurements in protected environments. It is particularly useful in remote sensing applications, as the device is insensitive to voltage drops over long lines due to its high impedance current output.

\subsection{Analog to Digital Converter (ADC)}

An analog to digital (A/D) converter is required to convert the analog signal from the temperature sensor into a digital signal for the microcontroller to read. For the purpose of reading the temperature of the fluid, a 12-bit microprocessor compatible A/D converter ICL7109 has been used since it is a high performance, CMOS, low power integrating analog to digital converter. The ICL 7109 operates at up to 30 conversions per second and provides the user with high accuracy, low noise $(15 \mu \mathrm{Vp}-\mathrm{p})$ output. Some of the features include true differential input and reference, drift of less than $1 \mu \mathrm{V} /{ }^{\circ} \mathrm{C}$, maximum input bias current of $10 \mathrm{pA}$, and typical power consumption of $20 \mathrm{~mW}$.

\subsection{Keypad}

The keypad for this application is a coded keyboard originally used for telephonic applications. These keypads are generally limited to 16 keys or fewer. The keypad interfaced to the microcontroller has 4 rows and 3 columns.

\section{HARDWARE IMPLEMENTATION}

All the hardware components, keypad, valves, temperature sensor, display unit have been interfaced with the microcontroller as shown in figure 3 . The programming has been done in the assembly language.

\subsection{Interfacing 4x3 Keypad}

Each of the rows and columns has one of the ends connected to the $5 \mathrm{~V}$ supply through $4.7 \mathrm{k} \Omega$ resistors. The other end is connected to the microcontroller port P0 pins P0.1 through P0.7. The key press is scanned by bringing each $\mathrm{X}$ row low in sequence and detecting a Y column low to identify each key in the matrix. The debounce time when the key is pressed or released is $20 \mathrm{~ms}$. The delay needed for key debounce is generated by a time delay loop.

\subsection{LCD Interfacing}

The 8 data lines of the display unit are connected to the microcontroller port P1 pins P1.0 through P1.7. Port pins P3.2, $\mathrm{P} 3.3$, and P3.4 of the microcontroller furnish the control signals register select (RS), read/write(R/W) and enable (E) respectively. Signal applied to the LCD's Enable (E) input clocks in the data. The $\mathrm{E}$ signal must be a positive going digital strobe, which is active while data and control information are true. The falling edge of the Enable signal enables the data/instruction register of the controller. The E signal is applied only when a specific module transaction is required. When the LCD controller is performing an internal operation the Busy Flag is set and will not accept any instruction. The Busy Flag is therefore checked before every instruction.

\subsection{Interfacing Temperature Sensing Unit}

AD590 temperature sensor, which is a semiconductor device, provides a current output proportional to the absolute temperature. This sensor has a nominal current sensitivity of $1 \mu \mathrm{A} / \mathrm{k}$. The current sensitivity is controlled by a potentiometer to give an output of $298.2 \mu \mathrm{A}$ at a temperature of $298.2 \mathrm{k}$. The output of the temperature sensor is sent to IN HI and IN LO pins of the A/D converter. Figure 4 shows the schematic for the temperature sensing unit.

\subsection{Interfacing A/D Converter}

Only 8-bits of the ADC are used to convert the analog temperature value to the corresponding digital value. With the analog output of the temperature sensor applied to pins IN HI and IN LO of the ADC, a reference voltage of $2 \mathrm{~V}$ is given to the $\mathrm{ADC}$ to begin the conversion. The chip enable pin CE/LOAD is grounded; bits $\mathrm{B} 1$ to $\mathrm{B} 8$ provide the digital output when the lower byte enable pin LBEN is enabled. The 8 data bits of the A/D converter are connected to port P2 pins P2.0 through P2.7 of the microcontroller. The analog signal is converted to digital data and is available at port 2 of AT89C51. This data is then stored in a register of the microcontroller and displayed on the LCD.

\subsection{Interfacing the Solenoid Valves}


The solenoid valves require actuation from the microcontroller so as to allow the liquid to flow through them. The solenoid valves used in this assembly require a $3 \mathrm{~V}, 400 \mathrm{~mA}$ dc supply. Since the output current of the microcontroller is only a few $\mu \mathrm{A}$, it is not capable of driving the valves. Hence a darlington array of two
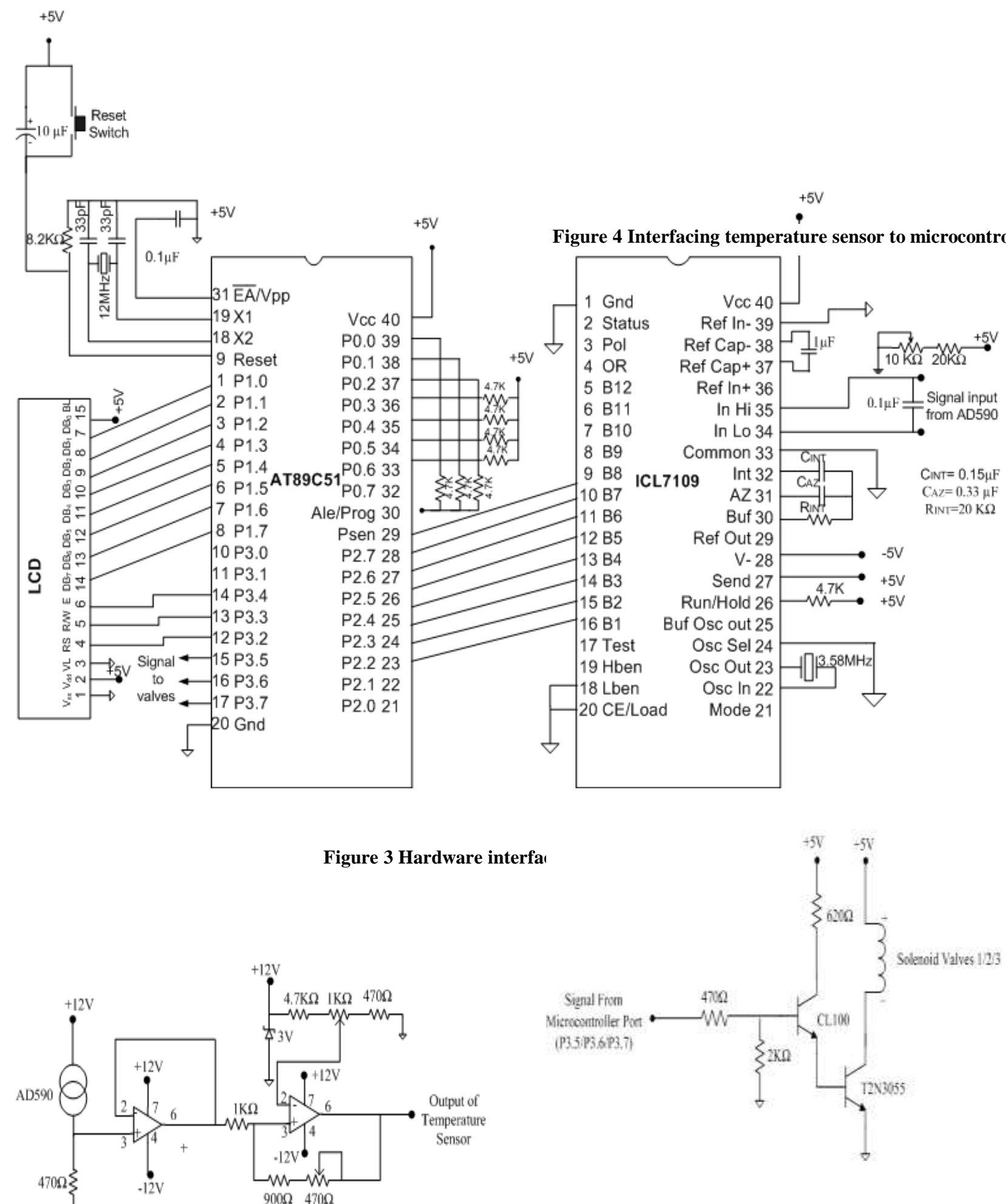

Figure 5 Darlington array for valve actuation

$900 \Omega 3$ 


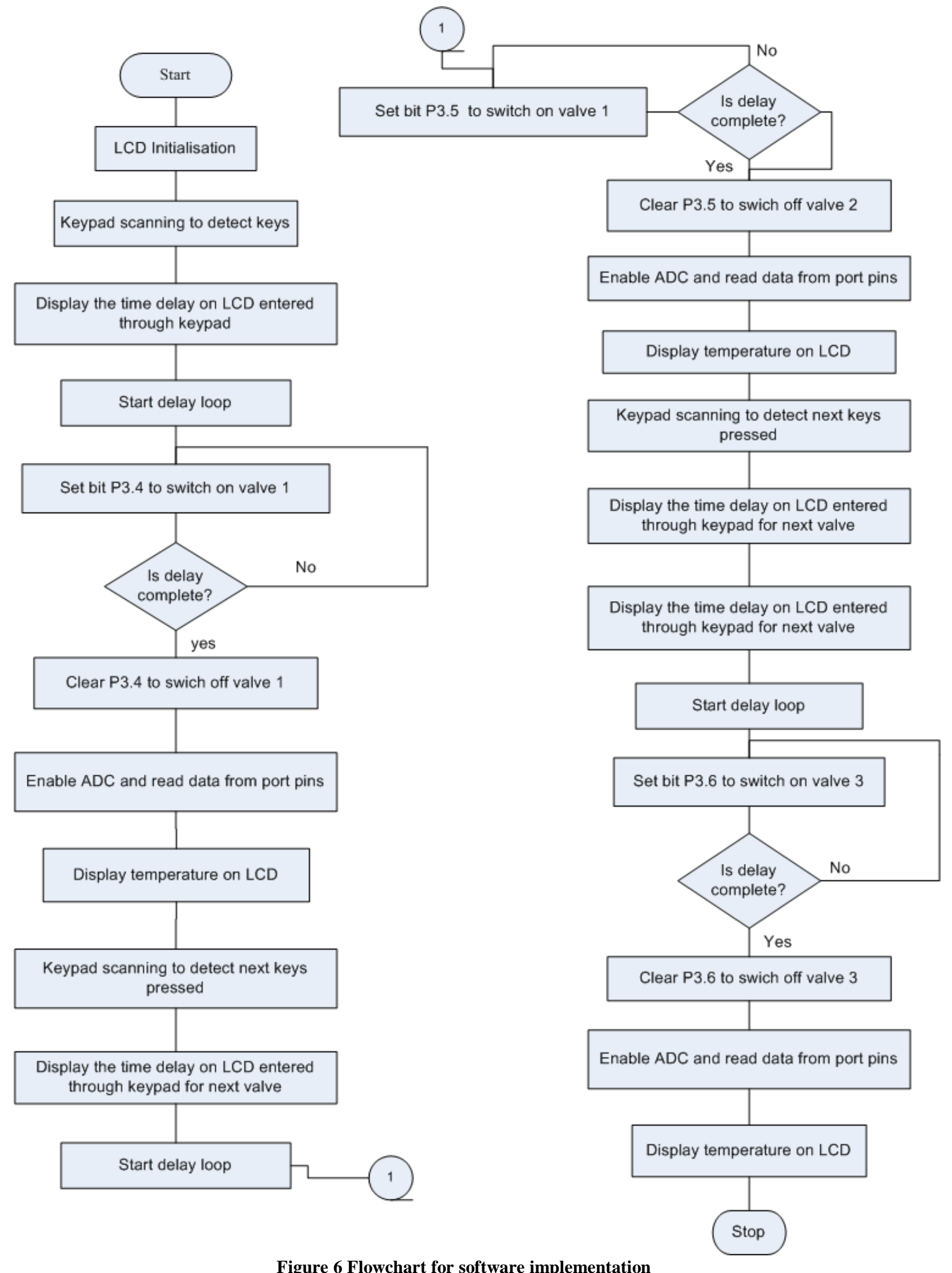


transistors, SL100 and a power transistor 2N3055, is used to provide the required current (figure 5). The valve actuation time is programmed through the keypad. Three port pins of the microcontroller P3.5, P3.6 and P3.7, separately provide actuation for each of the three valves. High signal on port pin P3.5 turns on valve 1, a high signal on P3.6 turns on valve 2 whereas high signal on P3.6 turns on valve 3. Only one port pin at a time provides a high signal to switch on the valve connected to it.

\section{SOFTWARE IMPLEMENTATION}

A dedicated assembly language program for controlling the hardware is written into the flash memory of the microcontroller. This program is first written at the command prompt which is then assembled using the assembler ASM51. The assembler checks the program for logical and syntax errors. Once the program has been checked for errors, a run command is issued that converts the assembled program into hex code. The microcontroller chip is then placed on a 40-pin flash programmer and the program is written into the flash memory in the form of a hex code.

The duration for valve actuation can be entered by the user using a 4x3 keypad. The microcontroller program checks whether any key on the keypad has been pressed. Once a valid key pressed has been detected, the valve is turned on for the duration ( in seconds) entered by the user. Time delay is generated using a program code. Once the time delay is complete, the microcontroller port provides a low signal that switches off the valve. This actuation time is displayed on line 1 of the LCD. The temperature sensor then senses the temperature and the $\mathrm{A} / \mathrm{D}$ converter converts the analog temperature into a digital data and the display unit then displays the temperature of the first buffer solution on line 2 of the LCD. Time delay for the next valve can then be entered. Once the second valve has been turned off, the actuation time for the third valve can then be entered. The flow chart for the software implementation is given in figure 6 .

\section{RESULTS}

The results obtained from the temperature sensor AD590 were compared with that of a digital thermometer. The ADC rounded off the analog signal from the temperature sensor into an 8-bit digital output to be displayed on the LCD. The output of AD590 was in millivolts $(\mathrm{mV})$ and was measured using a digital multimeter. The temperature sensor was calibrated to give $1 \mathrm{mV}$ signal corresponding to $1^{\circ} \mathrm{C}$ of temperature.

The performance of the flow assembly was investigated to examine the functionality of the valves interfaced to the microcontroller. The peristaltic pump pumped the specific buffer corresponding to the valves 1,2 and 3 onto the detector for the time periods specified for each valve entered through the keypad. The volume of buffer flow could be monitored by changing the flow rate of the pump.

\section{CONCLUSIONS}

A microcontroller based flow assembly was designed and fabricated. The system provided faster response as compared to manually operated flow assemblies. The results obtained from the ADC conformed to the actual temperature within the ambient temperature range. The flow assembly can be used at different flow rates ranging from $1.5 \mathrm{ml} / \mathrm{hr}$ to $156.5 \mathrm{ml} / \mathrm{hr}$ and its performance can be optimized by setting the flow rate according to the application. These automated flow assemblies offer the advantage of rapid sample throughput, small sample volumes, simplicity and minimal handling. Because of inherent sensitivity of certain detectors, FIA procedures hold great prospects in biological and biotechnological applications.

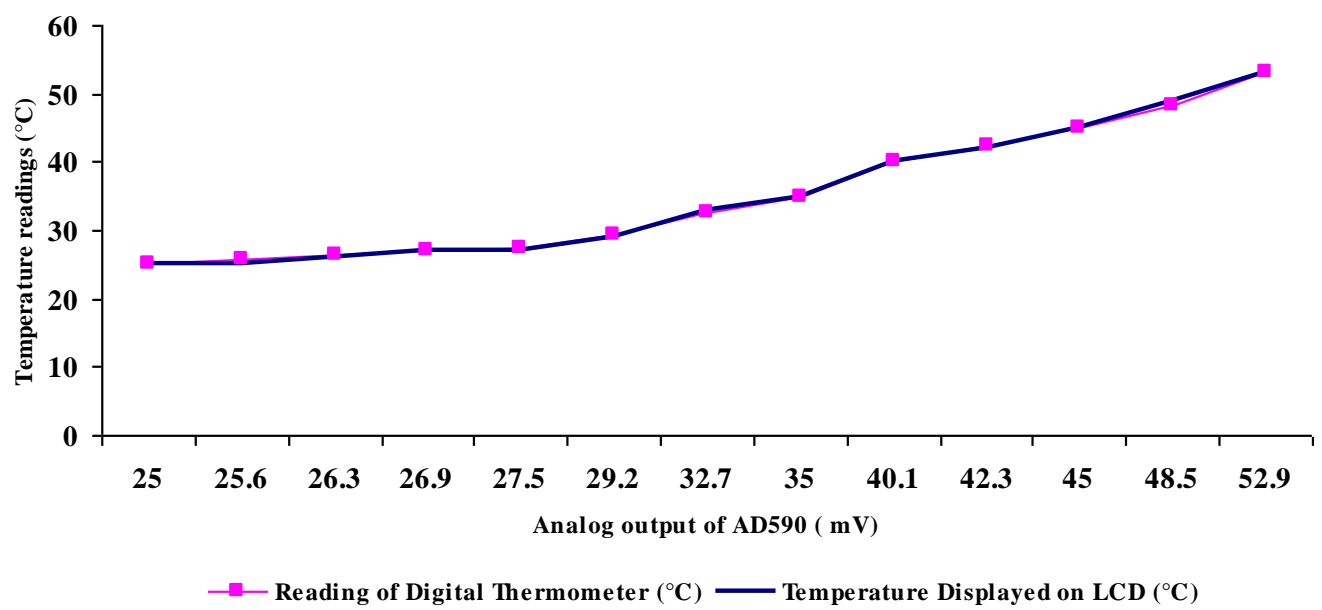

Figure 7 Graph comparing the readings of digital thermometer and ADC 


\section{REFERENCES}

[1] Koch, S., Wolf, H., Danapel, C., Feeler, K.A.2000. Optical flowcell multichannel immunosensor for the detection of biological warfare agents. Biosens. and Bioelect. 14 (1011):779-784.

[2] Roda, A., Girotti, S., Grigolo, B., Ghini, S., Carrea, G., Bovara, R., Zini, I.,Grimaldi, R.1991. Microdialysis and luminescent probe:Analytical and Clinical aspects. Biosens. and Bioelect. 6:21-29.

[3] Scheper,T., Buckman, A.F.1990. A fiberoptic biosensor based on fluorometric detection using confined macromolecular nicotinamide. Biosens. and Bioelect. 5:125135 .

[4] Simonian, A.L., Rainina, E.I., Fitzpatrick, P.F., Wild, J.R.1997. A tryptophan-2-monooxygenase based amperometric biosensor for L-tryptophan determination: use of a competitive inhibitor as a tool for selectivity increase. Biosens. and Bioelect.12:363-371
[5] Narang,U., Gauger, P., Anne, W., Ligler, F. 1998 Multianalyte detection using a capillary based flow immunosensor. Analytical Biochemistry 255:13-19.

[6] Lau, O., Shao,B., Lee, M. 2000. Affinity mass sensors: Determination of fructose. Analytica Chimica Acta 403: 49. 56.

[7] Chang, P., Shih, J. S. 2000. Multichannel piezoelectric quartz crystal sensor for organic vapours. Analytica Chimica Acta 403: 39-48.

[8] Bergamin, F., Zagalto, E., Krug, F.J., Keis, B.J. 1992. Ethanol fermentation monitoring by enzyamatic multi channel flow injection analysis. Analytica Chimica Acta 101:17-23.

[9] Ayala, K.J.2004. The 8051 Microcontroller, Architecture, Programming and Applications. New Delhi, India

[10] Ram, B. 1999. Fundamentals of Microprocessors and Microcomputers. New Delhi, India 